1 Department of Biochemistry, University of Leicester, The Adrian Building, University Road, Leicester LE1 7RH, UK

2 Department of Biosciences, University of Strathclyde, The Royal College, 204 George Street, Glasgow G1 $1 \mathrm{XL}$, UK

3 Department of Genetics, John Innes Centre Norwich NR4 7UH, UK

4 School of Biosciences, The University of Birmingham, Edgbaston, Birmingham, B15 2TT, UK

\section{Azole antifungals are potent inhibitors of cytochrome P450 mono-oxygenases and bacterial growth in mycobacteria and streptomycetes}

\author{
Kirsty J. McLean, ${ }^{1}$ Ker R. Marshall, ${ }^{1}$ Alison Richmond, ${ }^{2}$ lain S. Hunter, ${ }^{2}$
} Kay Fowler, ${ }^{3}$ Tobias Kieser, ${ }^{3}$ Sudagar S. Gurcha, ${ }^{4}$ Gurydal S. Besra ${ }^{4}$ and Andrew W. Munro ${ }^{1}$

Author for correspondence: Andrew W. Munro. Tel: +44 116252 3464. Fax: +44 1162523630. e-mail: awm9@le.ac.uk

The genome sequence of Mycobacterium tuberculosis has revealed the presence of 20 different cytochrome P450 mono-oxygenases (P450s) within this organism, and subsequent genome sequences of other mycobacteria and of Streptomyces coelicolor have indicated that these actinomycetes also have

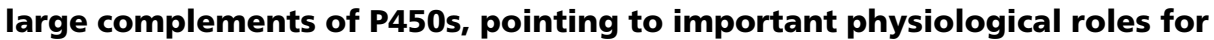
these enzymes. The actinomycete P450s include homologues of $14 \alpha$-sterol demethylases, the targets for the azole class of drugs in yeast and fungi. Previously, this type of P450 was considered to be absent from bacteria. When present at low concentrations in growth medium, azole antifungal drugs were shown to be potent inhibitors of the growth of Mycobacterium smegmatis and of Streptomyces strains, indicating that one or more of the P450s in these bacteria were viable drug targets. The drugs econazole and clotrimazole were most effective against $M$. smegmatis (MIC values of $<0.2$ and $0.3 \mu \mathrm{M}$, respectively) and were superior inhibitors of mycobacterial growth compared to rifampicin and isoniazid (which had MIC values of 1.2 and $36.5 \mu \mathrm{M}$, respectively). In contrast to their effects on the actinomycetes, the azoles showed minimal effects on the growth of Escherichia coli, which is devoid of P450s. Azole drugs coordinated tightly to the haem iron in $M$. tuberculosis H37Rv P450s encoded by genes Rv0764c (the sterol demethylase CYP51) and Rv2276 (CYP121). However, the azoles had a higher affinity for $M$. tuberculosis CYP121, with $K_{d}$ values broadly in line with the MIC values for $M$. smegmatis. This suggested that CYP121 may be a more realistic target enzyme for the azole drugs than CYP51, particularly in light of the fact that an S. coelicolor $\triangle C Y P 51$ strain was viable and showed little difference in its sensitivity to azole drugs compared to the wild-type. If the azole drugs prove to inhibit a number of important P450s in M. smegmatis and S. coelicolor, then the likelihood of drug resistance developing in these species should be minimal. This suggests that azole drug therapy may provide a novel antibiotic strategy against strains of $M$. tuberculosis that have already developed resistance to isoniazid and other front-line drugs.

Keywords: tuberculosis, azole drugs, CYP121, P450 inhibitors 


\section{INTRODUCTION}

The re-emergence of Mycobacterium tuberculosis (Mtb) as a worldwide threat to human health has been described by the World Health Organization (WHO) as having the capacity to cause a 'global catastrophe' (WHO fact sheet on tuberculosis at http://www.who. int/inf-fs/en/fact104.html). There are many reasons underlying the resurgence in $\mathrm{Mtb}$ infection rates around the world, but major factors include the synergy with the human immunodeficiency virus (HIV) (a large proportion of HIV-infected individuals, around $15 \%$, die ultimately from tuberculosis) and the development of drug-resistant and multi-drug-resistant strains of the pathogen, often as a result of poor or incomplete drug regimens in third-world countries (Heym et al., 1996). Regardless of the cause of its re-emergence, it is obvious that novel treatments are desperately required to prevent the further rapid spread of tuberculosis, which currently kills approximately 2 million people each year. It is estimated that between 2000 and 2020 nearly one billion people will become infected with Mtb, 200 million will become sick because of it and around 35 million will die from tuberculosis. At present, approximately one-third of the world's population is infected with tuberculosis, and the worldwide rate of new infections runs at one individual per second (WHO fact sheet on tuberculosis at http://www.who.int/inf-fs/en/fact104.html; Young \& Cole, 1993).

The determination of the genome sequence of $\mathrm{Mtb}$ (Cole et al., 1998) has provided a much needed boost for research into new drug targets against this pathogen. In particular, the vast array of genes involved in lipid metabolism (there are approximately five-fold the number of genes involved in lipid metabolism in $\mathrm{Mtb}$ compared with Escherichia coli, a bacterium with a similar genome size) is testament to the importance of this aspect of the organism's biochemistry to its viability and pathogenicity, particularly with regard to synthesis of the complex fatty acid, polyketide (and possibly sterol) components of its intricate outer envelope (Brennan \& Nikaido, 1995; Daffe \& Draper, 1998). These data suggest that targeting the lipid metabolism pathways of Mtb may provide an excellent route to attenuating or killing the bacterium. Indeed, only relatively recently has the biological target of the lead anti-Mtb drug isoniazid (isonicotinic acid hydrazide) been identified as enoyl-acyl carrier protein reductase, which is involved in fatty acid and mycolic acid synthesis (Blanchard, 1996). Isoniazid requires activation by the Mtb catalase/peroxidase enzyme to form a radical species that binds and covalently inactivates the NADHbinding site of the reductase (Saint-Joanis et al., 1999).

An unexpected finding from the Mtb genome-sequencing project was the unusually large component of cytochrome P450 mono-oxygenases (P450s) in the pathogen (Souter et al., 2000). Mtb encodes 20 P450s, whereas previous bacterial genomes had indicated that these haem-containing mono-oxygenase enzymes were relatively rare in bacteria. For instance, E. coli has none and Bacillus subtilis, with seven P450s, had the next largest bacterial complement of $\mathrm{P} 450$ s prior to determination of the Mtb genome sequence (Blattner et al., 1997; Kunst et al., 1997). This contrasts with the situation in eukaryotes, with the human genome promising to reveal up to $100 \mathrm{P} 450 \mathrm{~s}$, and the Arabidopsis thaliana genome encoding > 250 P450s (Lander et al., 2001; A. thaliana P450s site at http://drnelson.utmem. edu/Arab.genes.chr.html). The relatively large number of P450s in the Mtb genome indicates important physiological roles for these enzymes and, given that the substrate preference of the majority of $\mathrm{P} 450 \mathrm{~s}$ is for hydrophobic molecules, most are likely to be involved in lipid metabolism. An unexpected finding was that one of the Mtb P450s (the product of gene $R v 0764 c$ ) is a homologue of eukaryotic $14 \alpha$-sterol demethylases known as CYP51 (cytochrome P450 family 51) in the official P450 nomenclature system devised by Nelson et al. (1996). Previously, this type of P450 and the sterol biosynthetic pathway in which it participates were considered to be found exclusively in eukaryotes (Yoshida et al., 1997). However, while it remains uncertain as to whether Mtb actually has a sterol biosynthetic pathway, cholesterol has been reported to be present in the bacterial envelope (Lamb et al., 1998), and the Mtb CYP51 protein has been purified and shown to catalyse sterol demethylation, albeit at low rates (Bellamine et al., 1999). The genome sequence of the actinomycete Streptomyces coelicolor also shows a high number of P450-encoding genes, as well as marked similarities in genetic context (i.e. synteny) for a large proportion of the genome. However, specific biological roles for nearly all of the P450s in Mtb and S. coelicolor remain to be defined $[S$. coelicolor genome sequence home page at the Sanger Institute (http://www.sanger. ac.uk/Projects/S_coelicolor/)].

Recently, we have cloned and expressed the Mtb CYP51 enzyme and a second P450 from Mtb-CYP121, the product of the Rv2276 gene (Souter et al., 2000; McLean et al., 2002). We, and others, have demonstrated that CYP51 binds azole antifungal drugs tightly, as might be expected for a true homologue of the sterol demethylase class of P450. However, as we show here, it is also the case that CYP121 binds these azole drugs. Indeed, CYP121 binds these drugs with a higher affinity than CYP51, suggesting that CYP121 may be targeted by this type of drug in vivo. In studies of the effects of azole drugs on the growth of Mycobacterium smegmatis (which possesses a near identical complement of CYP genes to Mtb), we show that the azole antifungals are highly potent growth inhibitors, with very low MIC values. We also demonstrate that these drugs are effective as inhibitors of the growth of S. coelicolor and Streptomyces lividans, and compare their relative antibiotic effects with two CYP gene knockout variants of $S$. coelicolor. It appears that the actinomycetes may have at least two viable P450 targets for the azole antibiotic class (thus minimizing the opportunity for drug resistance), and that an azole-based strategy may be a new and effective means to combat Mtb. 


\section{METHODS}

Cloning and expression of CYP121. The CYP121 gene (Rv2276 from the Mtb genome project) encoding the CYP121 P450 was amplified from the Mtb chromosomal DNA cosmid library (cosmid number MTCY339) obtained from Professor Stewart Cole at the Pasteur Institute, Paris, France. Primers used in the reaction were designed from the $\mathrm{Mtb}$ genomic sequence (Cole et al., 1998): Forward primer, 5'-TATGACTCATATGACCGCGACCGTTCTGCTCG-3' ; Reverse primer, 5'-AAGACGGATCCTACCAGAGCACCGGAAGG-3'. In the forward primer, the bold letters indicate an engineered site for restriction enzyme $N d e$ I, incorporating the ATG initiation codon of CYP121. In the reverse primer, the bold letters indicate a BamHI site incorporating the TAG stop codon of CYP121. The PCR fragment generated from $R v 2276$ was cloned into plasmid vector pGEM-T (Promega), creating plasmid clone pKM2a, and its DNA sequence was verified by automated sequencing (Applied Biosystems DNA sequencer). The CYP121 gene was excised from pKM2a by digestion with NdeI and BamHI, and ligated into the T7 lac promoter vector pET11a (Novagen) that had been pre-digested with the same enzymes. Correctly ligated plasmids were selected by transformation of E. coli TG1 (Gibson, 1984) and restriction digests of plasmid preparations were made from transformant cultures. The expression plasmid was named pKM2b. All molecular techniques were performed using standard protocols (Sambrook et al., 1989).

The CYP121 enzyme was produced in the T7 RNA polymerase lysogen strain HMS174 (DE3) (Novagen). Conditions used for CYP121 production were growth of HMS174 (DE3)(pKM2b) transformants at $30^{\circ} \mathrm{C}$ in Terrific Broth (Sambrook et al., 1989) with vigorous agitation (250 r.p.m.) until the midexponential phase of growth $\left(\mathrm{OD}_{600} \sim 0 \cdot 5\right)$. The temperature was then decreased to $18{ }^{\circ} \mathrm{C}$, and the culture was induced with $100 \mu \mathrm{M}$ IPTG. Growth of the culture was continued for a further 20-24 h. After this time, cells were harvested by centrifugation $\left(7500 \mathrm{~g}, 30 \mathrm{~min}, 4^{\circ} \mathrm{C}\right)$, washed by resuspension in ice-cold $50 \mathrm{mM}$ Tris $/ \mathrm{HCl}(\mathrm{pH} 7 \cdot 2)$ plus $1 \mathrm{mM}$ EDTA (buffer A), pooled and recentrifuged. The final cell pellet was frozen at $-20{ }^{\circ} \mathrm{C}$ until use. CYP121 was purified from the culture (typically 5-8 l) by standard methods. For cell breakage, cells were thawed and resuspended in a small volume of buffer A containing protease inhibitors PMSF and benzamidine hydrochloride (both at $1 \mathrm{mM}$ ) and disrupted by sonication using a Bandelin Sonoplus GM2600 sonicator $(10 \times 20 \mathrm{~s}$ bursts at full power on ice, with appropriate cooling time between bursts). The extract was then passed through a French press [950 p.s.i. $(6.55 \mathrm{MPa}), 3$ passes] to complete the cell breakage process. The lysate was centrifuged at $18000 \mathrm{~g}$ for $30 \mathrm{~min}$ (at $4^{\circ} \mathrm{C}$ ) and the soluble extract was decanted. Protein was fractionated by ammonium sulfate precipitation (2 steps: $0-30 \%$ and $30-70 \%$ ), and the $30-70 \%$ pellet (containing CYP121) was retained. The pellet was resuspended in a minimal volume of buffer A containing $0.5 \mathrm{M}$ ammonium sulfate. The solution was then loaded onto a Phenyl-Sepharose column $(5 \times 30 \mathrm{~cm})$, which had been preequilibrated in buffer A containing $1.5 \mathrm{M}$ ammonium sulfate (buffer B). The protein was eluted in a linear gradient $(500 \mathrm{ml})$ of buffer B to buffer A. CYP121-containing fractions were dialysed against 5 lof buffer A, and loaded onto a Q-Sepharose column $(5 \times 25 \mathrm{~cm})$ that had been pre-equilibrated in buffer $A$. Protein was eluted in a linear gradient $(500 \mathrm{ml})$ of buffer A to buffer A plus $500 \mathrm{mM}$ potassium chloride. The CYP121containing fractions of highest purity were pooled and dialysed against 51 of $25 \mathrm{mM}$ potassium phosphate $(\mathrm{pH} 6.5$, buffer $\mathrm{C}$ ) before being loaded onto a hydroxyapatite column
$(5 \times 25 \mathrm{~cm})$ that had been pre-equilibrated in the same buffer. Protein was eluted in a linear gradient $(500 \mathrm{ml})$ of buffer $\mathrm{C}$ to $500 \mathrm{mM}$ potassium phosphate $(\mathrm{pH} \mathrm{6.5)}$. The fractions of highest purity $\left(A_{416} / A_{280}>1.5\right)$ were retained, pooled, concentrated by ultrafiltration (Centriprep 30; Millipore) to $<2 \mathrm{ml}$ and exchanged into buffer A plus glycerol $(50 \%, \mathrm{v} / \mathrm{v})$ by dialysis, prior to storage of the pure enzyme at $-80^{\circ} \mathrm{C}$.

Analysis of CYP121 purity, and drug binding to CYP121. Purity of the CYP121 protein was assessed by spectral properties (ratio of P450-specific absorption at $416.5 \mathrm{~nm}$ compared with protein-specific absorption at $280 \mathrm{~nm}$ ), and by SDS-PAGE of protein samples (on $10 \%$ polyacrylamide denaturing gels) at different stages in the purification process. The determination of the cytochrome P450 concentration was done from the Soret maximum of the oxidized enzyme in the resting (almost completely low-spin) state $\left(A_{\max }=416.5 \mathrm{~nm}\right)$, using the extinction coefficient $\varepsilon_{416.5}=95 \mathrm{mM}^{-1} \mathrm{~cm}^{-1}$, as described previously (Noble et al., 1999). Integrity of the protein was also verified by electrospray MS (Micromass platform quadruple mass spectrometer equipped with an electrospray ion source).

UV-visible absorption spectra were recorded on a Cary UV-50 Bio UV-visible scanning spectrophotometer using $1 \mathrm{~cm}$ pathlength quartz cells. Unless otherwise stated, spectra were recorded using approximately $5-10 \mu \mathrm{M}$ CYP121 in $50 \mathrm{mM}$ Tris $/ \mathrm{HCl}(\mathrm{pH} 7 \cdot 2)$. Binding of azole inhibitors to CYP121 was measured at $30^{\circ} \mathrm{C}$. The $\mathrm{P} 450$ inhibitors clotrimazole, econazole, ketoconazole, fluconazole, miconazole and 4-phenylimidazole were prepared as stock solutions (typically 10-25 mM) in DMSO. Small aliquots $(0 \cdot 1-0 \cdot 4 \mu \mathrm{l})$ corresponding to final concentrations of $1-100 \mu \mathrm{M}$ of inhibitor were added to a protein solution (typically $1-5 \mu \mathrm{M}$ P450), with the total addition less than $10 \mu \mathrm{l}$ inhibitor. Imidazole was made up in $50 \mathrm{mM}$ Tris $/ \mathrm{HCl}(\mathrm{pH} 7 \cdot 2)$ as a $3 \mathrm{M}$ stock solution and additions of up to $100 \mu \mathrm{l}$ were made to saturate CYP121. Spectra were recorded between 300 and $750 \mathrm{~nm}$ after each addition of substrate, and absorption shifts of the haem (Soret) absorption maximum from approximately $416.5 \mathrm{~nm}$ (azole ligand-free) to $423.5 \mathrm{~nm}$ (azole-saturated) were measured. $K_{\mathrm{d}}$ (binding constant) values for the binding of the azole ligands were determined by first generating difference spectra (by the subtraction of the original inhibitor-free spectrum from each subsequent inhibitor-bound spectrum). At this point, spectra were also multiplied by appropriate factors to account for dilution through addition of the azoles. Next, the maximum overall absorption change at each [azole] (peak absorption minus trough absorption in each difference spectrum) was plotted against the [azole]. The data points were then fitted to a rectangular hyperbola to generate the $K_{\mathrm{d}}$ value, using the equation $A=A_{\max }[\mathrm{I}] /\left(K_{\mathrm{d}}+[\mathrm{I}]\right)$, where $A$ is the observed shift in absorption at any given concentration of azole ligand [I], and $A_{\max }$ is the maximum overall shift in absorption at azole ligand saturation. For titrations in which the $K_{\mathrm{d}}$ value of the azole drug was not markedly higher than the [P450] used, data were fitted instead to a quadratic equation, which describes better the tight binding process observed and which accounts for the quantity of ligand complexed with the P450. The equation is $A=\left(\left(A_{\max } /(2 \mathrm{E})\right) \times\right.$ $\left.\left(\mathrm{I}+\mathrm{E}+K_{\mathrm{d}}\right)\right)-\left(\left(\mathrm{I}+\mathrm{E}+K_{\mathrm{d}}\right)^{2}-(4 \mathrm{IE})\right)^{0.5}$, where $A$ is the observed absorption difference at each azole addition, $A_{\max }$ is the maximal absorption difference at azole saturation, $\mathrm{E}$ is the total enzyme concentration and $\mathrm{I}$ is the azole inhibitor concentration used.

Determination of MIC values for $\boldsymbol{M}$. smegmatis. M. smegmatis mc $^{2} 155$ (Snapper et al., 1990) was used for testing the efficiency of the azole antifungal drugs. The MIC values for 
the azole antifungal drugs with $M$. smegmatis were measured by serial dilution of $M$. smegmatis cultures onto LB agar media (Sambrook et al., 1989) containing azole antifungal drugs (econazole, miconazole, fluconazole, ketoconazole or clotrimazole) in the concentration range $0 \cdot 01-250 \mu \mathrm{g} \mathrm{ml}^{-1}$. MICs were also determined for the validated anti-Mtb drugs isoniazid $\left(1-15 \mu \mathrm{g} \mathrm{ml}^{-1}\right)$ and metronidazole $\left(4-100 \mu \mathrm{g} \mathrm{ml}^{-1}\right)$. The MIC was defined as the minimal concentration of the drug required to completely inhibit the growth of $99 \%$ of the cells, as described previously (Cooksey et al., 1993; Phetsuksiri et al., 1999). All assays were performed in triplicate.

The effects of the azole antifungal drugs on the growth rate of M. smegmatis $\mathrm{mc}^{2} 155$ and E. coli TG1 were determined by growing overnight cultures of the bacteria in LB medium at $37^{\circ} \mathrm{C}$ with agitation at 180 r.p.m. The following morning, $50 \mu \mathrm{l}$ aliquots of the E. coli culture and $500 \mu \mathrm{l}$ aliquots of the M. smegmatis culture were transferred into tubes containing $5 \mathrm{ml}$ of fresh LB medium, and returned to the orbital incubator until the culture reached an $\mathrm{OD}_{600}$ value of $\sim 0 \cdot 1$ - this took approximately $1.5 \mathrm{~h}$ for E. coli and $3 \mathrm{~h}$ for M. smegmatis. At this stage, the antibiotics clotrimazole, econazole and isoniazid were added from the DMSO stock solutions (at final concentrations of 10,1 and $0 \cdot 1 \mu \mathrm{M}$, with DMSO at $\leqslant 0 \cdot 1 \%$, $\mathrm{v} / \mathrm{v}$ ) to separate tubes, and controls without drug addition, plus rifampicin $(5 \mu \mathrm{M})$ or plus DMSO $(0 \cdot 1 \%, \mathrm{v} / \mathrm{v})$ were added to further E. coli and M. smegmatis cultures. Growth of the cell cultures was then followed by $\mathrm{OD}_{600}$ measurements at 30-60 min intervals over the next 5-6 h (E. coli) or 10-12 h (M. smegmatis). The viability of the bacteria following antibiotic drug treatment was also assessed by measuring the growth rates of cultures of E. coli and M. smegmatis that had been pre-treated with econazole and isoniazid (at 10,1 and $0 \cdot 1 \mu \mathrm{M})$ and rifampicin $(5 \mu \mathrm{M})$ and restored to antibiotic-free medium. Inoculates $(50 \mu \mathrm{l}$ for E. coli, $500 \mu \mathrm{l}$ for $M$. smegmatis) were taken from the relevant drug-treated cultures $12 \mathrm{~h}$ after addition of the antibiotics, centrifuged briefly to pellet the cells, resuspended in the same volume of antibiotic-free medium and transferred to fresh LB medium for culture as before. The $\mathrm{OD}_{600}$ values of the two cultures were followed over the next several hours to assess bacterial recovery. All growth-rate measurements were done in triplicate.

Generation of CYP gene knockout mutants in S. coelicolor. A knockout mutant of the S. coelicolor A3(2) gene 3SCF60.06c (encoding putative P450 CYP105D5) was generated using the 'Redirect-Technology' PCR targeting system developed by Gust et al. (2002) under licence from Plant Bioscience Ltd, Norwich Research Park, Colney, Norwich, UK. This P450 (CYP105D5) shows closest similarity to the Mtb CYP121 enzyme at the amino acid level $(28 \%)$, as determined using the CLUSTAL W software at the European Bioinformatics Institute (http://www2.ebi.ac.uk/clustalw/).

A cassette comprising the apramycin-resistance gene aac(3)IV, the origin of transfer (oriT) from RK2 and flanking FLP recombinase recognition targets was modified by PCR to generate a gene-specific disruption cassette, using primers with $20 \mathrm{nt}$ identities to the ends of the cassette and $39 \mathrm{nt}$ identities to genomic regions flanking the $3 S C F 60.06 \mathrm{c}$ gene. The upstream primer was 5'-GTCCTTGTCGATGCCGATGTGCATGCCGGGAGCCTCCTATGTAGGCTGGAGCTGCTTC-3' and the downstream primer was 5'-ATCGATCCGACGCACTCCTCGTCTGCGAGGTCTTCCATGATTCCGGGGATCCGTCGACC-3'. S. coelicolor homologous sequences are shown in bold in both primer sequences. The linear disruption cassette DNA was then introduced into $E$. coli BW25113. This strain contained the temperature-sensitive $\lambda$ red recombination plasmid and the $S$. coelicolor cosmid
3F60. The recombination plasmid carries gam, the product of which inhibits host RecBCD exonuclease $\mathrm{V}$, thus allowing introduction of the linear disruption cassette into BW25113. The disruption cassette was allowed to recombine with the $3 S C F 60.06 \mathrm{c}$ gene on the $3 \mathrm{~F} 60$ cosmid at $30^{\circ} \mathrm{C}$, the recombination plasmid was then removed by incubation overnight at $37^{\circ} \mathrm{C}$ with concomitant selection of disrupted cosmid by incubation with $50 \mu \mathrm{g}$ apramycin $\mathrm{ml}^{-1}$ and $25 \mu \mathrm{g}$ kanamycin $\mathrm{ml}^{-1}$.

Prior to conjugative transfer of the mutant cosmid into $S$. coelicolor, the cosmid was first transformed into the methylation-deficient E. coli host ET12456, to prevent methylspecific restriction of the cosmid by S. coelicolor. ET12456 also contains the non-transmissible plasmid pUZ8002, which encodes the tra gene product that enables mobilization of vector DNA containing the RK2 oriT. Following intergeneric conjugative transfer into $S$. coelicolor, and selection in the presence of $50 \mu \mathrm{g}$ apramycin $\mathrm{ml}^{-1}$ and $25 \mu \mathrm{g}$ nalidixic acid $\mathrm{ml}^{-1}$ to selectively kill E. coli, colonies were screened twice for the presence of double-crossover recombinants by testing for resistance to apramycin and sensitivity to kanamycin on replica Difco nutrient agar plates. Finally, 3SCF60.06c knockout mutants were streaked onto mannitol soya flour medium plates (Hobbs et al., 1989) and stocks were prepared from single colonies.

An S. coelicolor knockout of the CYP51-encoding gene (SC7E4.20) was generated by transposon mutagenesis using Tn 4560, as described previously (Bhatt et al., 2002). Briefly, the strain was isolated from a screen of random transposon mutants generated using $\operatorname{Tn} 4560$, a derivative $(v p h$, viomycin resistance) of the Streptomyces fradiae Tn4556 for mutagenesis of S. coelicolor A3(2) (Bhatt et al., 2002). Tn4560 was introduced into $S$. coelicolor by intergeneric conjugation from E. coli [the non-methylating strain ET12567 containing the mobilizing plasmid pUZ8002 $\left(\mathrm{Km}^{\mathrm{r}}\right)$ and the transposon delivery vector pKay1, including Tn4560, $v p h$, plasmid markers $t s r$ and aac (3)IV, and oriT (RK2)]. Conjugation is described in detail elsewhere (Kieser et al., 2002). Random viomycin-resistant colonies were selected from the transposon library and the Tn4560 insertion site was determined by ligation-mediated PCR amplification (Bhatt et al., 2002) and dideoxy DNA sequencing. To facilitate PCR, the S. coelicolor DNA was digested completely using restriction enzyme EagI, which does not cut the Tn4560 ends. Then a non-phosphorylated, partially double-stranded adaptor was ligated (one strand only) to the EagI-generated fragments. PCR was then done using a Tn-specific primer and a primer specific for one strand of the adaptor, as described elsewhere (Fowler, 2002). DNA sequencing for one of the isolates showed that transposition had occurred within the SC7E4.20 gene, and this mutant was characterized further.

Assessment of effects of azoles on growth of Streptomyces strains. S. coelicolor A3(2), the two CYP knockout strains $(\triangle C Y P 51$ and $\triangle C Y P 105 D 5)$ and $S$. lividans TK24 were maintained on soya/mannitol agar slopes or as frozen spore suspensions (in distilled water) (Kieser et al., 2002). To assess the efficiency of the azole drugs on the growth of these strains, spore suspensions were thawed and spread over the surface of tryptone/soya agar (TSA; Oxoid) plates. At this stage, $6 \mathrm{~mm}$ antibiotic discs that had been pre-soaked in the relevant azole drug (or in isoniazid or rifampicin) or in DMSO or water (controls) were placed onto the surface of the plates. Cells were grown for 3 days at $30^{\circ} \mathrm{C}$, prior to determination of the antibiotic efficiency through measurement of the zone of cellular-growth inhibition created by each drug. A similar set of control experiments was performed using E. coli TG1. Cells 
were grown to an $\mathrm{OD}_{600}$ value of between $0 \cdot 7$ and $0 \cdot 8$, plated onto TSA medium and grown for $16-18 \mathrm{~h}$ at $30^{\circ} \mathrm{C}$, prior to analysis of growth inhibition. The incubation times allowed confluent bacterial cellular growth on the plates outside the zones of inhibition. MIC values in this system were defined as the minimum concentration of the drug required to give a measurable zone of inhibition around the disc. MIC values were also determined by serial dilution of S. coelicolor and S. lividans spore suspensions, and spotting these onto Difco Nutrient agar medium (Kieser et al., 2002). As with $M$. smegmatis, the MIC here was defined as the minimum drug concentration required to prevent growth of $99 \%$ of the cells. All assays were performed in triplicate.

Materials. Oligonucleotide primers for PCR were obtained from Perkin Elmer Applied Biosystems or were manufactured at the Protein and Nucleic Acid Laboratory (PNACL) at the University of Leicester, UK. All restriction enzymes and DNAmodifying enzymes were from New England Biolabs. Other modifying enzymes, Taq and Pfu DNA polymerase and T4 DNA ligase were obtained from Promega. Unless otherwise stated, all reagents used were obtained from Sigma and were of the highest grade available. Media and most solutions were made according to standard recipes (Sambrook et al., 1989). Tryptone/soya medium for the growth of Streptomyces spp. was from Oxoid. Econazole, ketoconazole and fluconazole were from ICN. Metronidazole, clotrimazole, miconazole and other azoles were from Sigma. Isoniazid was from Aldrich.

\section{RESULTS}

\section{Production and purification of CYP121}

The $R v 2276$ gene (hereafter referred to as CYP121) was amplified by PCR using Taq DNA polymerase as described in Methods, and verified to be of the correct size $(1 \cdot 188 \mathrm{~kb})$ by gel electrophoresis alongside DNA size standards ( $1 \mathrm{~kb}$ ladder; Gibco-BRL). The PCR product was cloned into pGEM-T (Promega), and successful clones were selected by $\alpha$-complementation on X-Gal- and IPTG-containing LB agar medium. The CYP121 gene was then excised from a verified clone (pKM2a), using NdeI and BamHI, and cloned into expression vector pET11a (Novagen) under the control of an IPTG-inducible T7 lac promoter, creating CYP121 expression plasmid pKM2b (Fig. 1).

Preliminary expression trials indicated that CYP121 was expressed to high levels in a variety of E. coli T7 RNA polymerase lysogen strains. However, large proportions of the CYP121 enzyme were found to form inclusion bodies. To achieve a high yield of soluble CYP121, the $E$. coli strain HMS174 (DE3) was used (Studier \& Moffat, 1986). A HMS174 (DE3)(pKM2b) transformant culture (typically of $5-10 \mathrm{l}$ ) was grown at $30^{\circ} \mathrm{C}$ until earlyexponential phase. Thereafter, mild IPTG induction $(100 \mu \mathrm{M})$ of the culture was used, and the growth temperature was reduced to $18{ }^{\circ} \mathrm{C}$. Cultivation was then continued for a further $24 \mathrm{~h}$. Under these conditions the overall expression level of CYP121 was reduced by approximately twofold (compared to growth and induction at $37^{\circ} \mathrm{C}$ ), but production of soluble CYP121 was greatly increased, allowing much higher recovery of soluble P450 from the cells.

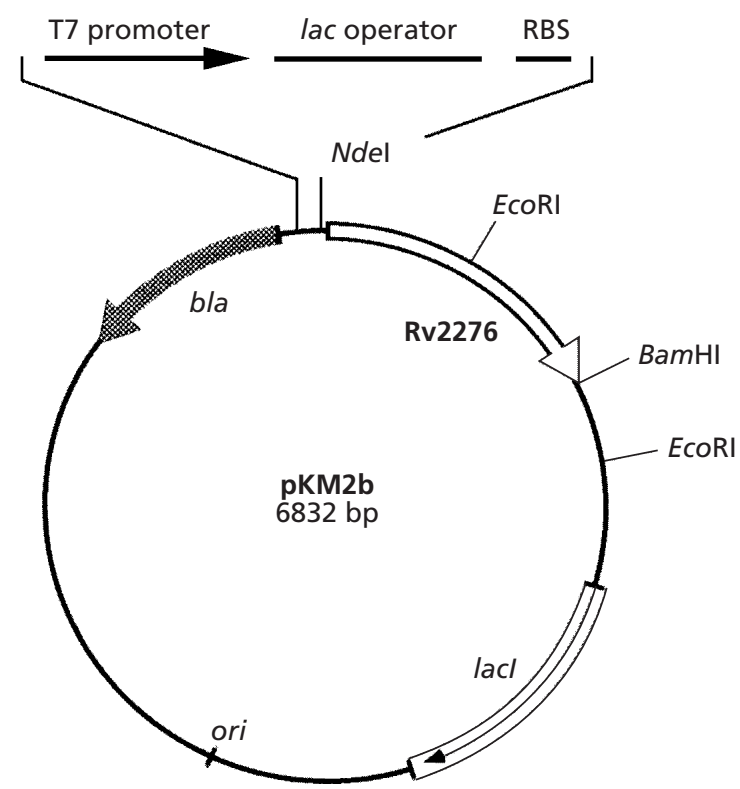

Fig. 1. Cloning of the CYP121 gene and creation of expression vector pKM2b. The CYP121 gene was cloned by PCR from a Mtb cosmid library, as described in Methods. The PCR product was cloned directly into pGEM-T (creating plasmid clone pKM2a), and then as a Ndel-BamHI fragment into plasmid vector pET11a, creating the CYP121-expressing plasmid pKM2b.

Typically, CYP121 was purified to homogeneity using ammonium sulfate fractionation followed by column chromatography using Phenyl-Sepharose, Q-Sepharose and hydroxyapatite. The relative purity of CYP121 was measured during its purification, by comparing the haem-specific absorption (at $416.5 \mathrm{~nm}$ ) with the total protein absorption at $280 \mathrm{~nm}$ at different stages of purification. These measurements typically showed that soluble CYP121 was purified approximately 50-fold from the original cell extract, and was recovered in yields of approximately $4 \mathrm{mg}$ pure $\mathrm{P} 450(1 \text { cell culture })^{-1}$. The overall yield of CYP121 was $\sim 20 \%$, based on recovery of haemoprotein. However, given the aforementioned tendency of the protein to misfold and form inclusion bodies, we suspect that much of the CYP121 present in the initial extract is haem-bound denatured enzyme, and that this is separated from the native form by ion exchange chromatography. The pure CYP121 bound carbon monoxide in its (dithionite-reduced) ferrous form, giving an absorption maximum at $448 \mathrm{~nm}$, typical of the P450 enzyme class (not shown). The ratio of haem-specific to total protein absorption (i.e. $A_{416 \cdot 5} /$ $A_{280}$ ) gives a measure of purity, with a value of approximately 1.9 indicating homogeneous CYP121, as verified by SDS-PAGE. Electrospray ionization MS indicated a single, intact species of molecular mass $43128 \mathrm{Da}$ (not shown). This correlated almost exactly with the predicted mass of CYP121 based on translation of its gene sequence, once the mass of the initiator methionine had been subtracted (43126 Da). A detailed description of the spectroscopic and biophysical proper- 

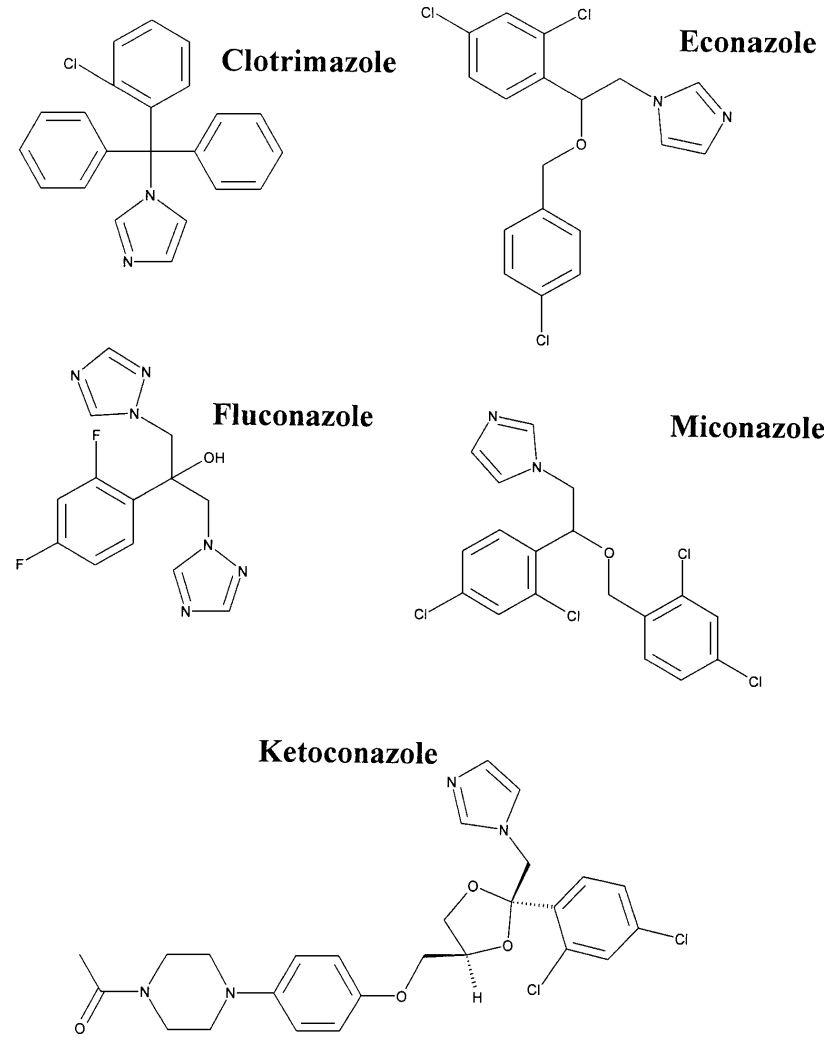

Fig. 2. Chemical structures of the azole antifungal drugs used in this study. In all cases, enzyme inhibition is achieved by ligation of basic nitrogen atoms from the inhibitors to the ferric haem iron of P450.

ties of CYP121 is presented elsewhere, and we have also determined the atomic structure of this P450 (McLean et al., 2002; D. Leys, C. G. Mowat, K. J. McLean, A. Richmond, S. K. Chapman, M. D. Walkinshaw, M. J. Sutcliffe \& A. W. Munro, unpublished data).

\section{Spectrophotometric analysis of azole drug binding to CYP121}

UV-visible absorption spectroscopy provides a simple and accurate method for the determination of the binding of substrates and inhibitors to P450s. The binding of azole inhibitors to P450s involves displacement of a water molecule (weakly ligated as an axial ligand to the haem iron) by a basic nitrogen from the azole ring of the inhibitor. This leads to a shift in the visible absorption spectrum of the P450, most notable through the shift of the major (Soret) absorption maximum to a longer wavelength. In the case of CYP121, the Soret band shifted from $416.5 \mathrm{~nm}$ (inhibitor-free) to $423 \cdot 5 \mathrm{~nm}$ (azole-saturated).

Each of the azole antifungal drugs tested here (clotrimazole, econazole, fluconazole, ketoconazole and miconazole) was found to bind tightly to CYP121, inducing a shift of the Soret spectral maximum to $423.5 \mathrm{~nm}$ (Fig. 2). The binding of clotrimazole, econazole and miconazole to CYP121 proved too tight to analyse accurately, even

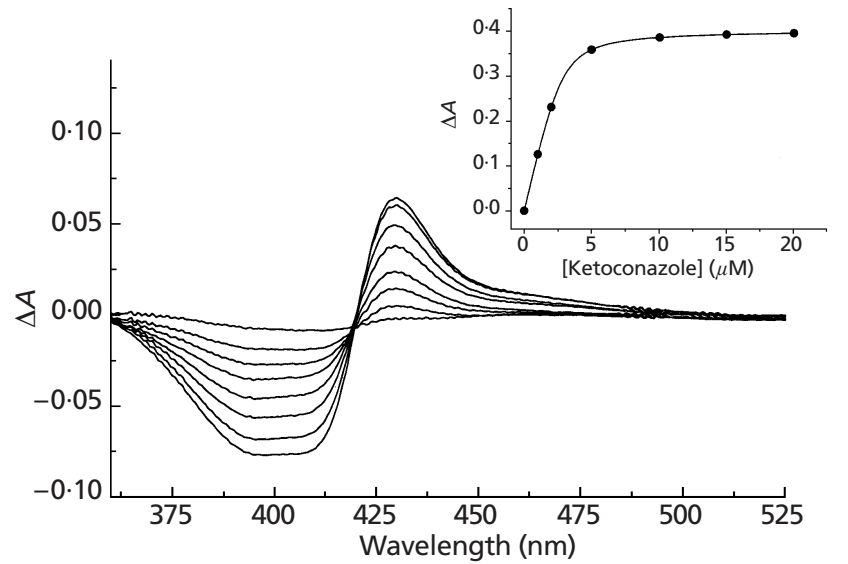

Fig. 3. Binding of ketoconazole to Mtb CYP121. The binding of the azole drug to the P450 haem induces shifts in the UV-visible absorption spectrum, due to replacement of the sixth water ligand to the ferric haem iron by the azole moiety of the drug. The main section of the figure shows a collection of difference spectra induced by the addition of the azole antifungal drug ketoconazole to oxidized CYP121 $(6.5 \mu \mathrm{M})$, with difference spectra generated by subtraction of the starting (azole-free) CYP121 absolute spectrum from those collected following addition of $0.2,1.2,2.2,3.2,5.2,7.2,13.2$ and $23.1 \mu \mathrm{M}$ ketoconazole. There is a progressive increase in the magnitude of the difference spectra with increasing ketoconazole concentrations. The azole drug complex is virtually fully formed by $23.1 \mu \mathrm{M}$, with the Soret absorption maximum shifted to $423.5 \mathrm{~nm}$ in the absolute spectrum. In the difference spectra, minima and maxima resulting from azole addition are located at approximately $395 / 408 \mathrm{~nm}$ and $430 \mathrm{~nm}$. The inset shows the plot of the maximal shifts in absorption data $\left(A_{430}\right.$ minus $\left.A_{408}\right)$ for binding of ketoconazole to CYP121 versus the relevant ketoconazole concentrations. Fitting the data to a quadratic function generates a $K_{d}$ value of $3 \cdot 3 \pm 0.3 \mu \mathrm{M}$ for the drug.

at low concentrations of CYP121 $(1 \cdot 2 \mu \mathrm{M})$. For these three azoles, the optical change associated with azole ligation occurred linearly with azole concentration, sharply reaching a plateau and indicative of stoichiometric binding to the P450. Even quadratic fits could not describe the $K_{\mathrm{d}}$ with sufficient accuracy. Evidently, the $K_{\mathrm{d}}$ value for these azoles is very low, and is likely to be $<0 \cdot 2 \mu \mathrm{M}$. Optical titrations with fluconazole revealed slightly weaker binding of this azole to CYP121, with a $K_{\mathrm{d}}$ value of $9 \cdot 7 \pm 0 \cdot 2 \mu \mathrm{M}$, as determined from a hyperbolic fit of the absorption change versus the fluconazole concentration. Ketoconazole bound to CYP121 with a slightly higher affinity than fluconazole, with a $K_{\mathrm{d}}$ value of $3 \cdot 3 \pm 0 \cdot 3 \mu \mathrm{M}$, as determined from the fit to the quadratic equation (Fig. 3).

The binding of imidazole $\left(K_{\mathrm{d}}=64 \pm 3 \mathrm{mM}\right)$ and 4phenylimidazole $\left(K_{\mathrm{d}}=53 \pm 3 \mu \mathrm{M}\right)$ to CYP121 was considerably weaker than that of clotrimazole, econazole or miconazole, indicating that the high affinity of the azole antifungals for CYP121 is determined primarily by favourable interactions between the bulky, polycyclic azole antifungals and the hydrophobic residues in the largely apolar active site of CYP121, rather than being driven by ligation of the azole group to the ferric haem iron. It is interesting to note that even the addition of a 
Table 1. Comparison of $M$. smegmatis MIC values for selected azole antifungal drugs with the relevant $K_{\mathrm{d}}$ values for Mtb CYP121 and CYP51

The MIC values for the azole antifungal drugs were determined as described in Methods. These values are compared with the relevant $K_{\mathrm{d}}$ values for the same drugs (derived from spectral titrations) for purified Mtb CYP121 (from this report) and the sterol demethylase CYP51 (Bellamine et al., 1999; Guardiola-Diaz et al., 2001). All MIC values have standard errors of $<10 \%$.

\begin{tabular}{|c|c|c|c|}
\hline \multirow[t]{2}{*}{ Drug } & \multirow{2}{*}{$\begin{array}{l}\text { MIC value for } M \text {. } \\
\text { smegmatis in } \mu \mathrm{M} \\
\quad\left(\text { and } \mu \mathrm{gl}^{-1}\right)\end{array}$} & \multicolumn{2}{|c|}{$K_{\mathrm{d}}$ value expressed in $\mu \mathrm{M}$ (and $\mu \mathrm{g} \mathrm{ml}^{-1}$ ) for } \\
\hline & & Mtb CYP121 & Mtb CYP51 \\
\hline Econazole* & $<0 \cdot 2(<0 \cdot 1)$ & $<0.2(<0.09)$ & $5 \cdot 4 \pm 1 \cdot 8 \neq(2 \cdot 4 \pm 0 \cdot 8)$ \\
\hline Clotrimazole* & $0 \cdot 3(0 \cdot 1)$ & $<0 \cdot 2(<0 \cdot 07)$ & $\sim 5 \ddagger(\sim 1 \cdot 7)$ \\
\hline Miconazole* & $2 \cdot 6(1 \cdot 25)$ & $<0 \cdot 2(<0 \cdot 10)$ & ND \\
\hline Ketoconazole* & $38(20)$ & $3 \cdot 3 \pm 0 \cdot 3(1 \cdot 75 \pm 0 \cdot 2)$ & 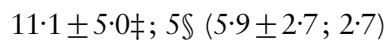 \\
\hline Fluconazole* & $>325(>100)$ & $9 \cdot 7 \pm 0 \cdot 2(3 \cdot 0 \pm 0 \cdot 1)$ & $\sim 10 \mathbb{S}(\sim 3 \cdot 1)$ \\
\hline Isoniazid $†$ & $36 \cdot 5(5)$ & $\mathrm{NC}$ & ND $\|$ \\
\hline Rifampicin $\dagger$ & $12(1)$ & $\mathrm{NC}$ & ND \\
\hline
\end{tabular}

ND, Not determined; NC, no spectral change observed.

*Antifungal drug.

† Anti-Mtb drug.

$\ddagger K_{\mathrm{d}}$ values for CYP51 are from Guardiola-Diaz et al. (2001).

\Estimated $K_{d}$ values from Bellamine et al. (1999).

\| Spectral changes were observed, but no $K_{\mathrm{d}}$ value was obtained (from Guardiola-Diaz et al., 2001).

hydrophobic phenyl moiety to the azoles (for 4-phenylimidazole cf. imidazole) is sufficient to reduce the $K_{\mathrm{d}}$ value by a factor of $10^{3}$. No significant spectral perturbations were observed on titration of CYP121 with the nitroimidazopyran drug metronidazole at concentrations up to $10 \mathrm{mM}$, suggesting that this drug binds more weakly to CYP121 than imidazole does.

\section{Determination of the efficiency of the azole antifungal drugs against $M$. smegmatis}

The MIC values for a range of azole antifungal drugs were determined using $M$. smegmatis, and these were compared with the MIC value for the leading anti-Mtb drug, isoniazid [isonicotinic acid hydrazide (INH)]. The MIC of isoniazid was $36.5 \mu \mathrm{M}\left(5 \mu \mathrm{g} \mathrm{ml}^{-1}\right)$. However, three of the five commercially available azole antifungal drugs proved more effective than isoniazid against $M$. smegmatis. The MIC values for clotrimazole $(0 \cdot 3 \mu \mathrm{M}$; $\left.0.1 \mu \mathrm{g} \mathrm{ml}^{-1}\right)$, miconazole $\left(2.6 \mu \mathrm{M} ; 1.25 \mu \mathrm{g} \mathrm{ml}^{-1}\right)$ and econazole $\left(<0.2 \mu \mathrm{M} ;<0 \cdot 1 \mu \mathrm{g} \mathrm{ml}^{-1}\right)$ indicated that all of these drugs were superior to isoniazid as inhibitors of M. smegmatis cell growth, and both clotrimazole and econazole were more effective than the potent anti-Mtb drug rifampicin $\left(\mathrm{MIC}=1 \cdot 2 \mu \mathrm{M} ; 1 \mu \mathrm{g} \mathrm{ml}^{-1}\right)$. Ketoconazole $\left(\mathrm{MIC}=38 \mu \mathrm{M} ; 20 \mu \mathrm{g} \mathrm{ml}^{-1}\right)$ was rather less effective than rifampicin, and fluconazole had a negligible effect on bacterial growth $\left(\mathrm{MIC}>325 \mu \mathrm{M} ;>250 \mu \mathrm{g} \mathrm{ml}^{-1}\right.$ ). DMSO, used as the solvent for the azoles, had no effect on cellular growth at the concentrations used $(<0 \cdot 1 \%)$. The order of potency of the azole drugs correlated with their $K_{\mathrm{d}}$ values for binding to the Mtb CYP121 enzyme (Table 1), suggesting that this P450 (rather than the
CYP51 sterol demethylase) may be the major site of action of these drugs.

The effects of the azole drugs on the growth rate of $M$. smegmatis were also determined, and the growth rates were compared with those of E. coli (see Methods). For E. coli, neither clotrimazole nor econazole (the two most effective azole drugs against $M$. smegmatis and S. coelicolor) had any significant effect on the specific growth rate (s.g.r.) of this organism when applied at concentrations of 10,1 and $0 \cdot 1 \mu \mathrm{M}$. Isoniazid (at the same concentrations) and DMSO also failed to decrease the growth rate of E. coli when compared with the control sample. In all cases the s.g.r. was around $0 \cdot 354 \pm 0 \cdot 044 \mathrm{~h}^{-1}$ [generation time (g.t.) $=0 \cdot 85 \pm 0 \cdot 10 \mathrm{~h}$ ] However, rifampicin at a concentration of $5 \mu \mathrm{M}$ completely prevented the growth of E. coli. In contrast to the results with E. coli, the azole drugs inhibited M. smegmatis growth strongly at low concentrations (Table 2). The naturally slower-growing control and DMSOtreated $M$. smegmatis samples had identical growth rates (s.g.r. $=0 \cdot 130 \pm 0 \cdot 008 \mathrm{~h}^{-1}$; g.t. $=2 \cdot 32 \pm 0 \cdot 14 \mathrm{~h}$ ), but growth was completely terminated by the presence of econazole at concentrations of 10 and $1 \mu \mathrm{M}$. At $0 \cdot 1 \mu \mathrm{M}$ econazole, the M. smegmatis s.g.r. was reduced by $54 \%$. Clotrimazole was also highly effective against M. smegmatis, but it was slightly less potent than econazole. At $10 \mu \mathrm{M}$ clotrimazole there was no $M$. smegmatis growth; at 1 and $0 \cdot 1 \mu \mathrm{M}$ clotrimazole the s.g.r. of M. smegmatis was reduced by 72 and $41 \%$, respectively. These azole drugs were far more effective than isoniazid, which decreased the growth rate of $M$. smegmatis by $26 \%$ only at the highest concentration tested $(10 \mu \mathrm{M})$. 
Table 2. Inhibition of $M$. smegmatis growth by azole antifungal drugs compared to inhibition of growth by anti-Mtb drugs

Growth of M. smegmatis cultures was recorded by measuring the $\mathrm{OD}_{600}$ value (see Methods). DMSO was added to the cultures at $0 \cdot 1 \%(\mathrm{v} / \mathrm{v})$ to act as a solvent for the drugs. Results are based on three replications and are shown \pm SD.

\begin{tabular}{|lcccc|}
\hline Drug & $\begin{array}{c}\text { Final concn } \\
(\boldsymbol{\mu} \mathbf{M})^{*}\end{array}$ & s.g.r. $\left(\mathbf{h}^{-\mathbf{1}}\right)$ & g.t. $(\mathbf{h})$ & $\begin{array}{c}\text { Decrease in } \\
\text { growth }(\%)\end{array}$ \\
\hline Control (no drug) & & $0 \cdot 130 \pm 0 \cdot 008$ & $2 \cdot 32 \pm 0 \cdot 14$ & - \\
Econazole† & 10 & $\mathrm{NG}$ & $\mathrm{NG}$ & 100 \\
& 1 & $\mathrm{NG}$ & $\mathrm{NG}$ & 100 \\
Clotrimazole† & $0 \cdot 1$ & $0 \cdot 060 \pm 0 \cdot 011$ & $5 \cdot 02 \pm 0 \cdot 92$ & 54 \\
& 10 & $\mathrm{NG}$ & $\mathrm{NG}$ & 100 \\
& 1 & $0 \cdot 036 \pm 0 \cdot 009$ & $8 \cdot 36 \pm 2 \cdot 09$ & 72 \\
Isoniazid $\ddagger$ & $0 \cdot 1$ & $0 \cdot 076 \pm 0 \cdot 004$ & $3 \cdot 96 \pm 0 \cdot 21$ & 41 \\
& 10 & $0 \cdot 096 \pm 0 \cdot 020$ & $3 \cdot 14 \pm 0 \cdot 65$ & 26 \\
Rifampicin $\neq$ & 1 & $0 \cdot 128 \pm 0 \cdot 008$ & $2 \cdot 35 \pm 0 \cdot 15$ & 0 \\
& $0 \cdot 1$ & $0 \cdot 123 \pm 0 \cdot 012$ & $2 \cdot 45 \pm 0 \cdot 24$ & 0 \\
& 5 & $0 \cdot 110 \pm 0 \cdot 011$ & $2 \cdot 74 \pm 0 \cdot 27$ & 15 \\
\hline
\end{tabular}

NG, No growth.

*A $1 \mu \mathrm{M}$ drug concentration corresponds to $0.45 \mu \mathrm{g} \mathrm{ml}^{-1}$ (econazole), $0.35 \mu \mathrm{g} \mathrm{m} l^{-1}$ (clotrimazole) or $0 \cdot 14 \mu \mathrm{g} \mathrm{ml}^{-1}$ (isoniazid). A $5 \mu \mathrm{M}$ concentration of rifampicin corresponds to $4 \cdot 1 \mu \mathrm{g}$ rifampicin $\mathrm{ml}^{-1}$.

† Antifungal drug.

$\neq$ Anti-Mtb drug.

Table 3. MIC values for azole antifungal drugs with S. coelicolor and S. lividans strains, as determined by the disc-based assay

DMSO, used as the solvent for the azole drugs, had no effect on the growth of the Streptomyces strains. The MIC values reported as molarities correspond to the following concentrations in units of $\mu \mathrm{g} \mathrm{ml} \mathrm{m}^{-1}$ for the MIC values with $S$. coelicolor: econazole, 2.2; clotrimazole, $3 \cdot 4$; miconazole, $3 \cdot 6$; ketoconazole, 130; fluconazole, 4600; metronidazole, $>17000$; isoniazid, $>14000$; rifampicin, 200000. All MIC values have standard errors of $<10 \%$.

\begin{tabular}{|c|c|c|c|c|}
\hline \multirow[t]{2}{*}{ Drug } & \multicolumn{4}{|c|}{ MIC values $(\mu \mathrm{M})$ for* } \\
\hline & $\begin{array}{c}\text { S. coelicolor } \\
\text { A3 }(2)\end{array}$ & $\begin{array}{c}\text { S. coelicolor } \\
\text { АCYP51 }\end{array}$ & $\begin{array}{c}\text { S. coelicolor } \\
\Delta C Y P 105 D 5\end{array}$ & $\begin{array}{l}\text { S. lividans } \\
\text { TK24 }\end{array}$ \\
\hline Econazole $†$ & $5(2)$ & $7 \cdot 5(2)$ & $7 \cdot 5(5)$ & $7 \cdot 5(5)$ \\
\hline Clotrimazole $†$ & $10(5)$ & $10(5)$ & $15(5)$ & $10(5)$ \\
\hline Miconazole† & $7 \cdot 5(5)$ & $7 \cdot 5(5)$ & $10(5)$ & $7 \cdot 5(5)$ \\
\hline Ketoconazole $†$ & $250(100)$ & $250(100)$ & $250(250)$ & $100(100)$ \\
\hline Fluconazole $†$ & $1.5 \times 10^{4}\left(1.5 \times 10^{4}\right)$ & $2 \times 10^{4}\left(1.5 \times 10^{4}\right)$ & $2 \times 10^{4}\left(2 \times 10^{4}\right)$ & $2 \times 10^{4}\left(2 \times 10^{4}\right)$ \\
\hline Metronidazole $\ddagger$ & $>10^{5}$ & $>10^{5}$ & $>10^{5}$ & $>10^{5}$ \\
\hline Isoniazid $\neq$ & $>10^{5}$ & $>10^{5}$ & $>10^{5}$ & $>10^{5}$ \\
\hline Rifampicin $\ddagger$ & $2 \cdot 5 \times 10^{5}$ & $2 \cdot 5 \times 10^{5}$ & $2 \cdot 5 \times 10^{5}$ & $2 \cdot 5 \times 10^{5}$ \\
\hline
\end{tabular}

*Values in parentheses are MIC values determined from colony growth on azole-containing medium.

† Antifungal drug.

$\neq$ Anti-Mtb drug.

Rifampicin at a concentration of $5 \mu \mathrm{M}$ caused only a $15 \%$ decrease in the M. smegmatis growth rate, a much lesser effect than observed when E. coli was grown in the presence of this drug.
To further examine the effects of the azole drugs on the viability of $M$. smegmatis, the growth of the bacterium post-azole treatment was measured. This experiment was performed using econazole (the most effective azole 


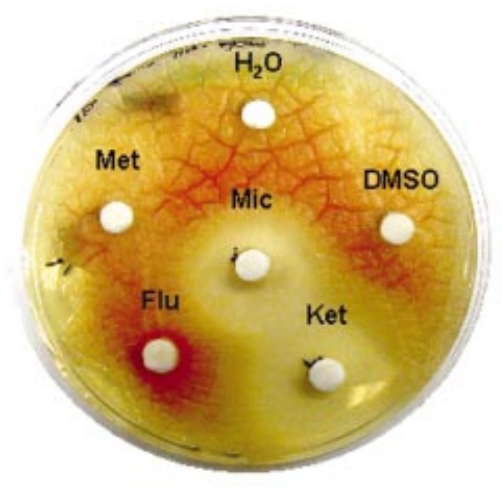

(a)

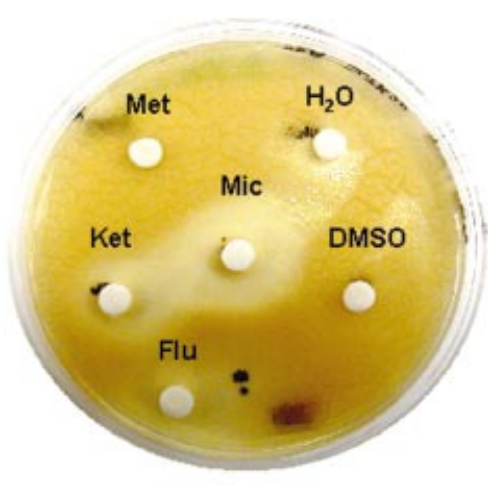

(b)
Fig. 4. Inhibition of Streptomyces cell growth by azole drugs. Azole antifungal drugs are potent inhibitors of the growth of $S$. coelicolor and S. lividans. The figure shows zones of inhibition of growth of S. coelicolor (a) and S. lividans (b) around discs soaked in the azole drugs miconazole (Mic), ketoconazole (Ket) and fluconazole (Flu), along with control discs soaked in metronidazole (Met; $100 \mathrm{mM}$ ), DMSO and water $\left(\mathrm{H}_{2} \mathrm{O}\right)$. S. coelicolor growth is inhibited by miconazole and ketoconazole (both at $10 \mathrm{mM}$ ), but not by fluconazole at the same concentration. S. lividans growth is also inhibited by miconazole and ketoconazole (both at $10 \mathrm{mM}$ ), and some inhibition is also observed for fluconazole at a higher concentration (100 mM). drug tested) and data for bacterial recovery compared with data obtained when isoniazid and rifampicin were used as the antibiotics. An identical set of experiments was performed on E. coli as a further control. For E. coli, $5 \mu \mathrm{M}$ rifampicin had a bactericidal effect, with complete inhibition of the growth of this organism upon the transfer of an inoculum of treated cells to antibioticfree medium. In contrast to the rifampicin pre-treatment, cells of E. coli pre-treated with either econazole or isoniazid (both at $10 \mu \mathrm{M}$ ) resumed growth, with rates identical to those of the untreated control cells (s.g.r. = $0 \cdot 354 \pm 0 \cdot 010 \mathrm{~h}^{-1}$; g.t. $=0 \cdot 85 \pm 0 \cdot 20 \mathrm{~h}$ ). For M. smegmatis cells pre-treated with isoniazid (at concentrations of 10,1 and $0 \cdot 1 \mu \mathrm{M}$ ) there was no significant difference in growth rates (s.g.r. $=0 \cdot 120 \pm 0 \cdot 010 \mathrm{~h}^{-1}$; g.t. $=2 \cdot 51 \pm$ $0 \cdot 21 \mathrm{~h}$ ) when compared to the untreated controls $\left(0 \cdot 124 \pm 0 \cdot 014 \mathrm{~h}^{-1} ;\right.$ g.t. $\left.=2 \cdot 43 \pm 0 \cdot 27 \mathrm{~h}\right)$. In contrast to the result with E. coli, cells of M. smegmatis pre-treated with rifampicin $(5 \mu \mathrm{M})$ recovered fully and resumed normal growth $\left(0 \cdot 126 \pm 0 \cdot 012 \mathrm{~h}^{-1}\right.$; g.t. $\left.=2 \cdot 39 \pm 0 \cdot 23 \mathrm{~h}\right)$. However, in M. smegmatis cells pre-treated with econazole there was a clear detrimental effect on bacterial growth in the subcultures, which was dependent on the initial concentration of the drug. In cells of M. smegmatis pre-treated with $10 \mu \mathrm{M}$ econazole, growth was reestablished at an s.g.r. of $0.069 \pm 0.012 \mathrm{~h}^{-1}$ (g.t. $=4 \cdot 36 \pm$ $0.76 \mathrm{~h})$; at $1 \mu \mathrm{M}$ econazole the s.g.r. was $0.097 \pm$ $0 \cdot 009 \mathrm{~h}^{-1}$ (g.t. $=3 \cdot 10 \pm 0 \cdot 29 \mathrm{~h}$ ), and at $0 \cdot 1 \mu \mathrm{M}$ econazole the s.g.r. was $0 \cdot 103 \pm 0 \cdot 008 \mathrm{~h}^{-1}$ (g.t. $=2 \cdot 92 \pm 0 \cdot 23 \mathrm{~h}$ ). Thus, after pre-treatment with $10 \mu \mathrm{M}$ econazole, the subcultured cells of M. smegmatis re-established growth at only $55 \%$ of the rate of the untreated control cells.

\section{Determination of the efficiency of the azole antifungal drugs against S. coelicolor A3(2) and S. lividans TK24}

As found with M. smegmatis, all of the azole antifungal drugs tested were potent inhibitors of cellular growth for S. coelicolor and S. lividans (Table 3; Fig. 4). MIC values, as determined from zones of inhibition around azole-soaked discs and from colony growth on azolecontaining medium (as described for M. smegmatis), were similar for both Streptomyces species, although the MIC values were generally slightly lower when determined by the colony-counting method. This may have resulted from the limited diffusion of the azole drugs through the agar medium in the disc-based assay.

The three azole drugs that bound most tightly to CYP121 and which were most effective against $M$. smegmatis in growth trials (i.e. econazole, clotrimazole and miconazole) were by far the most effective azoles against the Streptomyces species, with MIC values of $\leqslant 10 \mu \mathrm{M}$. The MIC values for the three azoles were all considerably lower than that for rifampicin. The next most effective azole, ketoconazole, had a similar antibacterial potency to rifampicin against the Streptomyces species. As seen with $M$. smegmatis, fluconazole was $<10^{3}$-fold as effective as econazole and clotrimazole against S. coelicolor and S. lividans. Isoniazid had a negligible effect on Streptomyces growth (MIC >100 mM), and the Mtb nitroimidazopyran drug candidate metronidazole also had a MIC value $>100 \mathrm{mM}$ with all of the Streptomyces strains tested. DMSO (the solvent for the drugs) also had no inhibitory effects on streptomycete growth at the concentrations tested.

In the disc-based assays of the azole and anti-Mtb drugs against $E$. coli TG1, none of the azole antifungal drugs were effective at concentrations up to $100 \mathrm{mM}$. Similarly, DMSO had no effect on E. coli growth. Metronidazole and isoniazid had only minor effects on the growth of E. coli TG1 (MIC values of 75 and $100 \mathrm{mM}$, respectively), but rifampicin was highly effective against this organism, with an MIC of $<5 \mu \mathrm{M}$.

At best, marginal differential effects on the growth of the two S. coelicolor cytochrome P450 knockout strains $(\triangle C Y P 51$ and $\triangle C Y P 105 D 5)$ were observed in comparison to the wild-type on exposure to the azole drugs. Any small difference in azole sensitivity was clearest in the $\triangle C Y P 105 D 5$ strain, where MIC values for the most effective azole drugs (econazole, clotrimazole and miconazole) were slightly higher than for the wild-type strain (Table 3). The most important feature to note regarding these strains was that the deletion of either P450 was non-lethal, and had little effect on the growth of the bacteria under laboratory conditions. Thus, 
neither CYP105D5 nor CYP51 is essential for viability under such conditions.

\section{DISCUSSION}

P450s are generally considered to be rare in bacteria, and to function mainly as components of dispensable catabolic pathways for the degradation of unusual carbon sources (Poulos et al., 1987; Peterson et al., 1992). Such enzymes would be effective targets for manipulation only under peculiar growth conditions. However, the recent genome-sequencing projects for $\mathrm{Mtb}$ (and other related mycobacteria) and $S$. coelicolor have indicated that these actinomycetes encode large numbers of P450s. There are 20 different $\mathrm{P} 450$ isoforms encoded within the Mtb genome [Cole et al., 1998; Mtb genome sequence home page at the Sanger Institute (http://www.sanger. ac.uk/Projects/M_tuberculosis/)], and the recent sequence of $S$. coelicolor indicates there to be $18 \mathrm{P} 450 \mathrm{~s}$ encoded within its genome (Bentley et al., 2002). The large numbers of P450s found in these actinomycetes have not (as yet) been found in other bacterial genera, and suggest that the P450s play several important physiological roles in mycobacteria and the streptomycetes; thus, they may provide novel drug targets against the pathogenic mycobacteria. In various Streptomyces species, roles for P450s are recognized in specific hydroxylations for the synthesis of polyketide antibiotics (e.g. P450picK in the synthesis of picromycin in Streptomyces venezuelae; Betlach et al., 1998). However, the roles of Mtb P450s remain to be elucidated, with the only possible exception being the putative sterol demethylase CYP51 (Souter et al., 2000; Bellamine et al., 1999; Lepsheva et al., 2001). The complexity of the lipids in the Mtb envelope may provide an explanation for the roles of several of the other P450s in this organism (Daffe \& Draper, 1998). The majority of the 20 P450s in Mtb are conserved in M. smegmatis and Mycobacterium bovis, with the notable exceptions of the homologues of those P450s encoded by Rv3121 and $R v 1256 c$, which are in regions of the genome deleted in the M. bovis BCG strain (RD12 and RD13, respectively) [The Institute for Genomic Research microbial genomes home page (http://www.tigr.org/tdb/mdb/mdbinprogress.html); M. bovis genome sequence home page at the Sanger Institute (http://www.sanger.ac.uk/ Projects/M_bovis/)]. Interestingly, the much smaller genome of Mycobacterium leprae (3.27 Mb cf. $4.44 \mathrm{Mb}$ in $\mathrm{Mtb}$ ) encodes only one functional P450, although there are several CYP pseudogenes retained within its genome $[M$. leprae genome sequence home page at the Sanger Institute (http://www.sanger.ac.uk/Projects/ M_leprae/)]. The sole viable CYP gene remaining in M. leprae is ML2088, or CYP118, whose closest relative in $\mathrm{Mtb}$ is the CYP140 protein encoded by the $R v 1880 \mathrm{c}$ gene (34\% amino acid sequence identity). Obviously, the characterization of this particular P450 isoform may shed light on a potentially critical P450-mediated role retained in $M$. leprae, a bacterium which has undergone dramatic gene decay during its evolution (Cole et al., 2001).
Azole drugs are commonly used in the treatment of fungal and yeast infections, and act primarily by inhibiting the activity of the P450 CYP51, which is a lanosterol demethylase involved in the generation of ergosterol, a critical sterol for the cellular membrane of yeasts and fungi (Schuster, 1993). Fungal resistance to azole drugs is frequently associated with CYP51 mutations that reduce affinity of the altered proteins for the drugs (Aoyama et al., 2000). A rationale for the use of azoles as antibacterials has been absent, due to assumptions that bacteria generally do not possess sterol biosynthetic pathways. However, a CYP51-like enzyme has been characterized in Mtb and this enzyme has been shown to catalyse the demethylation of lanosterol and a plant sterol (obtusifoliol), as would be expected for a bona fide CYP51 enzyme (Bellamine et al., 1999). The subsequent determination of the atomic structure of the Mtb CYP51 in complex with the drug fluconazole provided the first molecular description of the mode of interaction of an azole antifungal drug with a P450 enzyme. The structure is thus of great importance in understanding how the specificity for the azole is determined, and may enable de novo design of more selective and potent azole inhibitors of CYP51. However, azole antifungals are used mainly as topically applied antibiotics for the treatment of superficial yeast and fungal infections. Due to their cross-reactivity (often with micromolar or higher affinity) with human P450 isoforms, many of the azole drugs are not systemically tolerated and are toxic when administered orally or by intravenous injection (Zhang et al., 2002). Much research has gone into the production of systemically tolerated azole drugs, and fluconazole has been among the most successful of the generation of triazole drugs that is now in clinical use. Research remains active in this area, and voriconazole is a good example of a newly developed systemically tolerated triazole with an improved potency and spectrum of antifungal activity compared to fluconazole (Hoffman \& Rathbun, 2002). In view of the fact that we have demonstrated that several azole drugs are powerful inhibitors of mycobacterial (and streptomycete) growth, there would appear to be good evidence for the development and use of these drugs as anti-Mtb agents.

The fact that azole antifungal drugs are highly effective as inhibitors of the growth of M. smegmatis and of Streptomyces strains does not lead us immediately to the identification of their major intracellular target enzyme(s). However, it would appear unlikely that the drugs do not target $\mathrm{P} 450$ (s) in vivo (particularly in light of their tight binding to Mtb CYP121 and CYP51), and their ineffectiveness against E. coli (which is devoid of P450s) suggests that these drugs have negligible effects on other cellular enzymes, including the respiratory cytochromes. However, any assumption that the sole P450 target in mycobacteria and streptomycetes is the sterol demethylase homologue CYP51 is clearly invalid based on this study. We present data that show that not only does the Mtb CYP121 enzyme bind tightly to azole antifungal drugs, but that its $K_{\mathrm{d}}$ values for the azole 
drugs tested are almost invariably lower than the respective values for Mtb CYP51, and correlate better with the MIC values determined for the inhibition of $M$. smegmatis than do the respective CYP51 $K_{d}$ values (Table 1). We have recently determined the atomic structure of Mtb CYP121, and find that (among the known P450 atomic structures) it most closely resembles the P450eryF (CYP107A1) enzyme from Saccharopolyspora erythraea, which is involved in the synthesis of the polyketide antibiotic erythromycin (CuppVickery et al., 1996). P450eryF was shown to have strong affinity for azole antifungal drugs, and its structure has also been determined in the ketoconazolebound form (Cupp-Vickery et al., 2001). This demonstrates that the azole drugs may also effectively inhibit the P450s involved in biotransformations of polycyclic polyketides. Our data indicate that there are at least two P450s in Mtb which bind tightly to azole antifungal drugs, and that the MIC values against $M$. smegmatis correlate much more closely with the $K_{\mathrm{d}}$ values for CYP121 than for CYP51. Thus, we consider CYP121 to be a more likely target for the azole drugs in mycobacteria. The fact that a CYP51 knockout is non-lethal (and has little effect on azole sensitivity) in the related actinomycete $S$. coelicolor lends further weight to this argument. In our more recent work on a third Mtb P450 (CYP140, the product of the $R v 1880 c$ gene) we have found that this P450 also binds the azole antifungal drugs, albeit with a rather lower affinity than CYP121 does (K. R. Marshall, J. Clapp, K. J. McLean \& A. W. Munro, unpublished data). The fact that each of the first three Mtb P450s that we have characterized shows high affinity for azole drugs suggests that a number of the remaining $17 \mathrm{P} 450$ isoforms present in $\mathrm{Mtb}$ could also bind drugs of this class tightly. Thus, it is possible that the potent effects of the azole drugs on M. smegmatis and other actinomycetes are a result of them targeting several important P450s. Obviously, the development of resistance to drugs is likely to be much less of a problem if the antibiotic used has multiple cellular targets.

Gene-knockout experiments indicate that the S. coelicolor CYP51 gene (isolated by transposon mutagenesis) is non-essential for bacterial viability under laboratory growth conditions, and a strain lacking a functional CYP51 gene grew at a rate comparable with the wildtype strain. The CYP51 homologue in yeasts and fungi is the target enzyme for the azole drugs in these organisms, and is a critical enzyme in maintaining membrane integrity. Although purified Mtb CYP51 has been shown to demethylate dihydrolanosterol and the plant sterol obtusifoliol at low rates (Bellamine et al., 1999), the physiological function of this enzyme remains unclear. It appears unlikely that the streptomycete CYP51 plays such a critical role in vivo as the fungal CYP51s do, due to the viability of the $\triangle$ CYP51 strain. Similarly, deletion of the CYP105D5 gene was also non-lethal - this gene encodes the S. coelicolor P450 with highest similarity (at the amino acid level) to Mtb CYP121. However, the rather low level of amino acid sequence identity between CYP105D5 and CYP121 (28\%) indicates that these proteins are unlikely to be direct homologues, although both align most closely with polyketide-metabolizing P450s from Streptomyces spp. The CYP gene knockout strains of $S$. coelicolor A3(2) generally have similar azole drug sensitivity to the wild-type strain and to S. lividans TK24, but the MIC values for the azoles are very low in all cases. Econazole appears to be the most effective azole (MIC as low as $2 \mu \mathrm{M} ; 0.9 \mu \mathrm{g} \mathrm{ml}^{-1}$ ), followed by clotrimazole $\left(5 \mu \mathrm{M} ; 1.7 \mu \mathrm{g} \mathrm{ml}^{-1}\right)$ and miconazole $(5 \mu \mathrm{M}$; $\left.2 \cdot 4 \mu \mathrm{g} \mathrm{ml}^{-1}\right)$. Ketoconazole is less effective $(100 \mu \mathrm{M}$; $52 \mu \mathrm{g} \mathrm{ml}^{-1}$ ) than these three azoles, and fluconazole is considerably less effective ( $\left.15 \mathrm{mM} ; 4.6 \mathrm{mg} \mathrm{m}^{-1}\right)$ than them. All of the azole drugs were far more effective at inhibiting the growth of the Streptomyces strains than the anti-tubercular drugs isoniazid and rifampicin, and the candidate anti-tubercular drug metronidazole (Table $3)$. However, the azole drugs were even more potent inhibitors of the growth of M. smegmatis, a closer relative of $\mathrm{Mtb}$ and a convenient model organism for the pathogen. The M. smegmatis MIC values for econazole and clotrimazole are more than 10 -fold lower than those for the Streptomyces strains $(<0 \cdot 2$ and $0 \cdot 3 \mu \mathrm{M}$, respectively, compared to 5 and $10 \mu \mathrm{M}$, respectively, for $S$. coelicolor) and those for miconazole and ketoconazole are two- to threefold lower. With regard to M. smegmatis, the validated anti-tubercular drugs isoniazid and rifampicin are far more effective as growth inhibitors of this organism (cf. Streptomyces strain), but econazole, clotrimazole and miconazole still have much lower MIC values than isoniazid ( $>180$-fold in the case of econazole) and both econazole and clotrimazole remain at least four-fold more effective in inhibiting growth than rifampicin (Tables 1 and 3). Moreover, M. smegmatis cells pre-treated with econazole show retarded growth when inoculated into antibiotic-free medium, whereas cells similarly pre-treated with isoniazid or rifampicin re-establish growth with negligible differences in their s.g.r. values compared to untreated cells.

There is some irony in the fact that fluconazole (one of the very few systemically tolerated azole drugs) is quite ineffective against M. smegmatis and Streptomyces strains. However, this does point to the fact that there is clearly an element of selectivity that can be used to target the Mtb P450s specifically. The available structure of the CYP51-fluconazole complex, and our own forthcoming high-resolution atomic structure of the CYP121 enzyme, will provide the basis by which molecular modelling, in consort with synthetic chemistry, can be used to develop superior azole-based inhibitors, which have the potential to be more effective anti-Mtb drugs. The superior pharmacokinetic properties and higher potency of the newly developed drug voriconazole over, for example, fluconazole is testament to the fact that high levels of selectivity for individual P450 isoforms can be achieved; this process can only be aided by the availability of atomic structures of target P450s in the pathogen Mtb.

Novel Mtb drug targets are desperately needed due to the prevalence of strains of the pathogen that are 
resistant to the 'traditional' drugs, most notably to antibiotics such as rifampicin, isoniazid, ethionamide and ethambutol (e.g. Heym et al., 1996). Our preliminary work shows that the azoles are highly effective in preventing the growth of M. smegmatis and related Streptomyces strains. The azole drugs are known to be strong inhibitors of fungal growth, and it may be assumed that their target enzyme in the fungi (CYP51) is also that inactivated in the actinomycetes. However, it is important to recognize that the bacterial CYP51s remain non-validated as a target for these drugs. Indeed, the fact that a CYP51 knockout in S. coelicolor is non-lethal and has no considerable effect on the growth or sensitivity of this organism to azole drugs suggests that it may not be a target at all in bacteria. With such a plethora of P450s encoded by Mtb (20) and S. coelicolor (18), there are potentially a number of distinct drug-target P450s. The polyketide hydroxylase P450eryF (one of the most highly related P450s to CYP121) has high affinity for the azole drug ketoconazole $\left(K_{\mathrm{d}}=\sim 3 \mu \mathrm{M}\right)$, making obvious the fact that both polyketide- and sterol-metabolizing P450s can exhibit high affinity for azoles (Cupp-Vickery et al., 2001). This may be an important factor in understanding the apparent potency of the tested azole drugs as inhibitors of the growth of the actinomycetes tested. While both the mycobacteria and the streptomycetes have large complements of P450s, there are few close P450 homologues between these two bacterial groups at the amino acid level. This may reflect different physiological functions for many of these enzymes. For instance, the synthesis of polyketide antibiotics by the streptomycetes may account for the majority of the P450s in these organisms, whereas Mtb may have uses for several P450s in the metabolism of complex envelope lipids. Despite a similar number of encoded P450s in $S$. coelicolor (18) and Mtb (20), it should be noted that the $S$. coelicolor genome $(8 \cdot 67 \mathrm{Mb})$ is approximately twice the size of that of $\mathrm{Mtb}(4 \cdot 41 \mathrm{Mb})$. Thus, there is more than double the P450 'density' in the Mtb genome than in the S. coelicolor genome. This again points to genusspecific roles for the P450s and suggests that a higher proportion of the $\mathrm{Mtb} \mathrm{P} 450 \mathrm{~s}$ are involved in cell envelope lipid (as opposed to antibiotic) transformations, given the relative importance of lipid metabolism in the physiology of the pathogen (Cole et al., 1998). The fact that lipid metabolism (in all its forms) is more critical to Mtb and other mycobacteria than to streptomycetes may also be suggested by the fact that isoniazid is so ineffective against Streptomyces strains (Table 3). In $\mathrm{Mtb}$, the pro-drug isoniazid is activated by a catalase/peroxidase enzyme (KatG); the free radical product binds to the $\mathrm{NAD}^{+}$-sites of enoyl-acyl carrier protein reductase (InhA) and 3-oxo-acyl-acyl carrier protein synthase (KasA) to inactivate these enzymes, which are essential for mycolic acid synthesis (Wengenack \& Rusack, 2001). All of these enzymes are present in S. coelicolor, but isoniazid is $>2700$-fold more effective against M. smegmatis than the Streptomyces strains tested (Tables 1 and 3). Regardless of their distinct cellular functions, it appears clear that there are P450s which are essential for viability and/or growth in both streptomycetes and mycobacteria, and that these can be inactivated by azole drugs at very low concentrations. This clearly raises the exciting prospect of the development of new drug therapies for strains of $\mathrm{Mtb}$ that have developed resistance to traditional therapies, particularly if the azoles prove to have multiple P450 targets in Mtb.

In future work, we intend to identify the natural substrate for CYP121, by performing binding studies using extracts from Mtb and/or from infected human cells. We are also investigating the redox partners for CYP121 and the other Mtb P450 systems. To this end, we have recently produced and purified the ferredoxinreductase-like protein FprA (product of the $R v 3106$ gene), and we are also characterizing potential ferredoxin-encoding genes from $\mathrm{Mtb}$. We are also examining the effectiveness of azole drugs on the growth and viability of the more clinically relevant mycobacteria M. bovis (BCG) and Mtb. Our long-term aim is to define the structure of and the drug selectivity in these pathogen P450 systems, to reconstitute turnover systems with the native redox partners and to establish those Mtb P450s that can provide new drug targets in the ongoing war against multi-drug-resistant strains of Mtb.

\section{ACKNOWLEDGEMENTS}

The authors would like to thank the BBSRC, EPSRC, MRC and EU (Project 'X-TB') for their financial support for these studies. We are also grateful to Professor Stewart Cole (Institut Pasteur, Paris, France) for provision of cosmid DNA and scientific discussions. G.S.B. is currently a Lister InstituteJenner Research Fellow. We would like to thank Dr Tobias Ost (Dept of Chemistry, University of Edinburgh, UK) for recording the mass spectrum of CYP121.

\section{REFERENCES}

Aoyama, Y., Kudo, M., Asai, K., Okonogi, K., Horiuchi, T., Gotoh, O. \& Yoshida, Y. (2000). Emergence of fluconazole-resistant sterol 14-demethylase P450 (CYP51) in Candida albicans is a model demonstrating the diversification mechanism of P450. Arch Biochem Biophys 379, 170-171.

Bellamine, A., Mangla, A. T., Nes, W. D. \& Waterman, M. R. (1999). Characterization and catalytic properties of the sterol 14alpha-demethylase from Mycobacterium tuberculosis. Proc Natl Acad Sci US A 96, 8937-8942.

Bentley, S. D., Chater, K. F., Cerdeno-Tarraga, A.-M. \& 40 other authors (2002). Complete genome sequence of the model actinomycete Streptomyces coelicolor A3(2). Nature 417, 141-147.

Betlach, M. C., Kealey, J. T., Ashley, G. W. \& McDaniel, R. (1998). Characterization of the macrolide P-450 hydroxylase from Streptomyces venezuelae which converts narbomycin to picromycin. Biochemistry 37, 14937-14942.

Bhatt, A., Stewart, G. R. \& Kieser, T. (2002). Transposition of Tn4560 of Streptomyces fradiae in Mycobacterium smegmatis. FEMS Microbiol Lett 206, 241-246.

Blanchard, J. S. (1996). Molecular mechanisms of drug resistance in Mycobacterium tuberculosis. Annu Rev Biochem 65, 215-239.

Blattner, F. R., Plunkett, G., 3rd, Bloch, C. A. \& 14 other authors (1997). The complete genome sequence of Escherichia coli K-12. Science 277, 1453-1474. 
Brennan, P. J. \& Nikaido, H. (1995). The envelope of the mycobacteria. Annu Rev Biochem 64, 29-63.

Cole, S. T., Brosch, R., Parkhill, J. \& 39 other authors (1998). Deciphering the biology of Mycobacterium tuberculosis from the complete genome sequence. Nature 393, 537-544.

Cole, S. T., Eiglmeier, K., Parkhill, J. \& 41 other authors (2001). Massive gene decay in the leprosy bacillus. Nature 409, 10071011.

Cooksey, R. C., Crawford, J. T., Jacobs, W. R., Jr \& Shinnick, T. M. (1993). A rapid method for screening antimicrobial agents for activities against a strain of Mycobacterium tuberculosis expressing firefly luciferase. Antimicrob Agents Chemother 37, 1348-1352.

Cupp-Vickery, J. R., Han, O., Hutchinson, C. R. \& Poulos, T. L. (1996). Substrate-assisted catalysis in cytochrome P450eryF. Nat Struct Biol 3, 632-637.

Cupp-Vickery, J. R., Garcia, C., Hofacre, A. \& McGee-Estrada, K. (2001). Ketoconazole-induced conformational changes in the active site of cytochrome P450eryF. J Mol Biol 311, 101-110.

Daffe, M. \& Draper, P. (1998). The envelope layers of mycobacteria with reference to their pathogenicity. Adv Microb Physiol 39, 131-203.

Fowler, K. (2002). Transposon Mutagenesis of Streptomyces coelicolor A3(2). PhD thesis. University of East Anglia, UK.

Gibson, T. J. (1984). Studies in the Epstein-Barr Virus Genome. $\mathrm{PhD}$ thesis. University of Cambridge, UK.

Guardiola-Diaz, H. M., Foster, L.-A., Mushrush, D. \& Vaz, A. D. N. (2001). Azole-antifungal binding to a novel cytochrome P450 from Mycobacterium tuberculosis : implications for the treatment of tuberculosis. Biochem Pharmacol 61, 1463-1470.

Gust, B., Kieser, T. \& Chater, K. F. (2002). Redirect (C) Technology: PCR-Targeting System in Streptomyces coelicolor. Norwich, UK: John Innes Centre.

Heym, B., Philipp, W. \& Cole, S. T. (1996). Mechanisms of drug resistance in Mycobacterium tuberculosis. Curr Top Microbiol Immunol 215, 49-69.

Hobbs, G., Frazer, C. M., Gardner, D. C. J., Cullum, J. A. \& Oliver, S. G. (1989). Dispersed growth of Streptomyces in liquid culture. Appl Microbiol Biotechnol 31, 272-277.

Hoffman, H. L. \& Rathbun, R. C. (2002). Review of the safety and efficacy of voriconazole. Expert Opin Investig Drugs 11, 409-429.

Kieser, T., Bibb, M. J., Buttner, M. J., Chater, K. F. \& Hopwood, D. A. (2002). Practical Streptomyces Genetics. Norwich, UK: John Innes Foundation.

Kunst, F., Ogasawara, N., Moszer, I. \& 48 other authors (1997). The complete genome sequence of the gram-positive bacterium Bacillus subtilis. Nature 390, 249-256.

Lamb, D. C., Kelly, D. E., Manning, N. J. \& Kelly, S. L. (1998). A sterol biosynthetic pathway in Mycobacterium. FEBS Lett 437 , 142-144.

Lander, E. S., Linton, L. M., Birren, B. \& 253 other authors (2001). Initial sequencing and analysis of the human genome. Nature 409 , 860-921.

Lepesheva, G. I., Podust, L. M., Bellamine, A. \& Waterman, M. R. (2001). Folding requirements are different between sterol 14alphademethylase (CYP51) from Mycobacterium tuberculosis and human or fungal orthologs. J Biol Chem 276, 28413-28420.

McLean, K. J., Cheesman, M. R., Rivers, S. L. \& 9 other authors (2002). Expression, purification and spectroscopic characteriz- ation of the cytochrome P450 CYP121 from Mycobacterium tuberculosis. J Inorg Biochem (in press).

Nelson, D. R., Koymans, L., Kamataki, T. \& 9 other authors (1996). P450 superfamily: update on new sequences, gene mapping, accession numbers and nomenclature. Pharmacogenetics 6, 1-42.

Noble, M. A., Miles, C. S., Chapman, S. K., Lysek, D. A., Mackay, A. C., Reid, G. A., Hanzlik, R. P. \& Munro, A. W. (1999). Roles of key active-site residues in flavocytochrome P450 BM3. Biochem J 339, 371-379.

Peterson, J. A., Lu, J. Y., Geisselsoder, J., Graham-Lorence, S., Carmona, C., Witney, F. \& Lorence, M. C. (1992). Cytochrome P-450terp. Isolation and purification of the protein and cloning and sequencing of its operon. J Biol Chem 267, 14193-14203.

Phetsuksiri, B., Baulard, A. R., Cooper, A. M., Minnikin, D. E., Douglas, J. D., Besra, G. S. \& Brennan, P. J. (1999). Antimycobacterial activities of isoxyl and new derivatives through the inhibition of mycolic acid synthesis. Antimicrob Agents Chemother 43, 1042-1051.

Poulos, T. L., Finzel, B. C. \& Howard, A. J. (1987). High-resolution crystal structure of cytochrome P450cam. J Mol Biol 195, 687-700.

Saint-Joanis, B., Souchon, H., Wilming, M., Johnsson, K., Alzari, P. M. \& Cole, S. T. (1999). Use of site-directed mutagenesis to probe the structure, function and isoniazid activation of the catalase/peroxidase, KatG, from Mycobacterium tuberculosis. Biochem J 338, 753-760.

Sambrook, J., Fritsch, E. F. \& Maniatis, T. (1989). Molecular Cloning: a Laboratory Manual, 2nd edn. Cold Spring Harbor, NY : Cold Spring Harbor Laboratory.

Schuster, I. (1993). Ergosterol synthesis: the major target on antimycotic therapy. In Medicinal Implications in Cytochrome P450 Catalysed Biotransformations, pp. 147-185. Edited by K. Ruckpaul \& H. Rein. Berlin: Akademie Verlag.

Snapper, S. B., Melton, R. E., Mustafa, S., Kieser, T. \& Jacobs, W. R., Jr (1990). Isolation and characterization of efficient plasmid transformation mutants of Mycobacterium smegmatis. Mol Microbiol 4, 1911-1919.

Souter, A., McLean, K. J., Smith, W. E. \& Munro, A. W. (2000). The genome sequence of Mycobacterium tuberculosis reveals cytochromes P450 as novel drug targets. J Chem Technol Biotechnol 75, 933-941.

Studier, F. W. \& Moffat, B. A. (1986). Use of bacteriophage T7 RNA polymerase to direct selective high-level expression of cloned genes. J Mol Biol 189, 113-130.

Wengenack, N. L. \& Rusnak, F (2001). Evidence for isoniaziddependent free radical generation catalysed by Mycobacterium tuberculosis KatG, and the isoniazid-resistant mutant KatG(S315T). Biochemistry 40, 8990-8996.

Yoshida, Y., Noshiro, M., Aoyama, Y., Kawamoto, T., Horiuchi, T. \& Gotoh, O. (1997). Structural and evolutionary studies on sterol 14-demethylase P450 (CYP51), the most conserved P450 monooxygenase: II. Evolutionary analysis of protein and gene structures. J Biochem 122, 1122-1128.

Young, D. B. \& Cole, S. T. (1993). Leprosy, tuberculosis, and the new genetics. J Bacteriol 175, 1-6.

Zhang, W. J., Ramamoorthy, Y., Kilicarslan, T., Nolte, H., Tyndale, R. F. \& Sellers, E. M. (2002). Inhibition of cytochromes P450 by antifungal imidazole derivatives. Drug Metab Dispos 30, 314-318.

Received 16 May 2002; revised 21 June 2002; accepted 9 July 2002. 